\title{
Modeling Vortex Swarming In Daphnia
}

\section{Journal Article}

\section{Author(s):}

Mach, Robert; Schweitzer, Frank

Publication date:

2007

Permanent link:

https://doi.org/10.3929/ethz-b-000005386

Rights / license:

In Copyright - Non-Commercial Use Permitted

Originally published in:

Bulletin of Mathematical Biology 69(2), https://doi.org/10.1007/s11538-006-9135-3 


\title{
Modeling Vortex Swarming In Daphnia
}

\author{
Robert Mach, Frank Schweitzer* \\ ETH Zurich, Chair of Systems Design, Kreuzplatz 5, CH-8032 Zurich
}

Received: 21 July 2005 / Accepted: 27 April 2006 / Published online: 19 August 2006

(C) Society for Mathematical Biology 2006

\begin{abstract}
Based on experimental observations in Daphnia, we introduce an agentbased model for the motion of single and swarms of animals. Each agent is described by a stochastic equation that also considers the conditions for active biological motion. An environmental potential further reflects local conditions for Daphnia, such as attraction to light sources. This model is sufficient to describe the observed cycling behavior of single Daphnia. To simulate vortex swarming of many Daphnia, i.e. the collective rotation of the swarm in one direction, we extend the model by considering avoidance of collisions. Two different ansatzes to model such a behavior are developed and compared. By means of computer simulations of a multi-agent system we show that local avoidance-as a special form of asymmetric repulsion between animals-leads to the emergence of a vortex swarm. The transition from uncorrelated rotation of single agents to the vortex swarming as a function of the swarm size is investigated. Eventually, some evidence of avoidance behavior in Daphnia is provided by comparing experimental and simulation results for two animals.
\end{abstract}

Keywords Active motion · Swarming · Zooplankton · Brownian agents

\section{Introduction}

Swarming is a prominent example of complex behavior in biological systems. This form of collective motion may emerge from the interplay of individual behavior and local interactions of a large number of individuals (agents). Swarms (also called herds, flocks, schools) can often be observed in certain mammals, fish, insects, and birds for various benefits, such as enhanced feeding and mating as well as more successful predator avoidance (see e.g. Huth and Wissel, 1992, 1994; Parrish and Hamner, 1997; Parrish and Edelstein-Keshet, 1999; Couzin and Krause, 2001);

\footnotetext{
* Corresponding author.

E-mail address: fschweitzer@ethz.ch (Frank Schweitzer)
} 
(Parrish et al., 2002; Okubo and Levin, 2002). This has been reported for several prey animals, e.g. in planktivore fish (Partridge, 1982; Hall et al., 1986), in some species of birds (Caraco et al., 1980), as well as in zooplankton (Jakobsen et al., 1994; Kvam and Kleiven, 1995).

Detailed experimental investigations on swarming, however, are rare, either because of the size of the animals or because well defined conditions for experiments are difficult to realize. Earlier chance observations were reported for horizontally circling zooplankton in the field (Lobel and Randall, 1986). They triggered further experiments with Daphnia relevant for the current paper (see also Sections 2 and 6). It has been shown that under certain circumstances single Daphnia circle horizontally around a vertical artificial light shaft to which they are attracted. For high Daphnia densities a swarm emerges, where all Daphnia circle in the same randomly chosen direction (Ordemann, 2002; Ordemann et al., 2003a,b).

The physical, biological, and chemical reasons for swarming in Daphnia in particular and in prey animals in general are not completely understood. Biological considerations suggest that circling is the least energy consuming motion for permanently moving animals to stay as a group at a certain local position without frequently bumping into each other. Recent studies concentrated on the response of individuals as well as groups to various external influences, such as available food, food gradients and predator threat (Jakobsen and Johnsen, 1987; Larsson and Kleiven, 1995; Kleiven et al., 1996; Larsson, 1997; Jensen, 2000). In particular, Øien (2004) used methods from plasma kinetic theory to derive macroscopic equations-so-called fluid-dynamic equations-for the density of Daphnicle (Daphnia-like particle) swarms as a function of food-concentration, food saturation of Daphnia and a threat field of predators. The advantage of this approach is, that these equations can be solved analytically by approximations in linear space. However, it is difficult to compare these results for swarming to rotating real Daphnia swarms, as we are interested in.

While Øien (2004) concentrates his description on the macroscopic level (density ond velocity destributions of the swarm), we aim at understanding swarming from a "microscopic" approach. That means, we derive equations of motion for individual entities - so-called agents - and investigate the collective motion by means of a multi-agent system. This allows us to understand the behavior at the system level from the interactions of the entities comprising the system.

Individual-based or agent-based modeling has turned out to be a very useful tool for modeling biological phenomena at various levels of organization (Grünbaum and Okubo, 1994; Deutsch, 1999; Flierl et al., 1999; Kunz and Hemelrijk, 2003). Thus, recently different computer architectures have been developed to simulate the collective behavior of interacting agents in distributed artificial intelligence (see e.g. http: / / www . swarm. org/). However, due to their rather complex simulation facilities many of these simulation tools lack the possibility to investigate systematically and in depth the influence of specific interactions and parameters. Instead of incorporating only as much detail as is necessary to produce a certain emergent behavior, they put in as much detail as possible, and thus reduce the chance to understand how emergent behavior occurs and what it depends on. Therefore, in this paper, we follow a different multi-agent approach, based on 


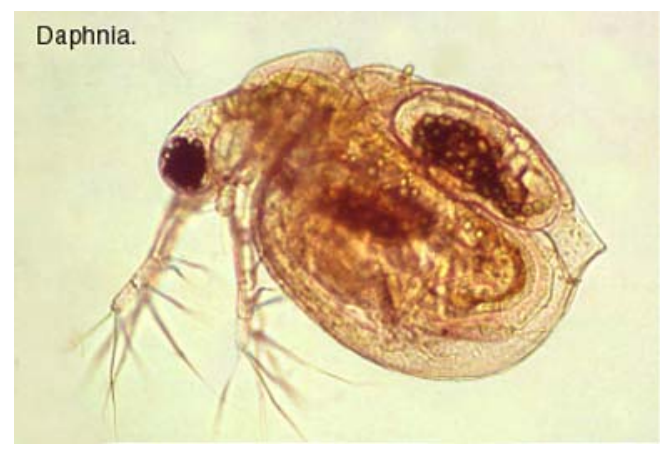

Fig. 1 Daphnia, Courtesy of Stephen Durr.

Brownian agents (Schweitzer, 2003) (see Section 3), that-in addition to its computational suitability - can be also investigated by means of analytical methods from statistical physics and mathematics.

The objective of the present study is to investigate the requirements on the microscopic level that lead to the formation of a vortex swarm on the macroscopic level. A swarm is called vortex swarm if animals cycle around an imaginary axis in the same rotational direction. On the global level (i.e. in three dimensions) one observes the emergence of a cone. As the main (cycling) motion takes place in the horizontal plane, we have restricted both the experimental observations and our simulation to two dimensions for simplicity. To find out more about physical reasons for vortex swarming, we first summarize some experimental observations previously reported, and then set-up a "minimalistic" multi-agent model to test some biologically relevant assumptions that may lead to the observed swarming behavior.

\section{Experimental observations on Daphnia motion}

In this section, we summarize some biological facts about Daphnia animals and their collective movement which have been reported in the literature. This information shall be used to motivate our swarming model in the following sections.

The water flea or Daphnia (see Fig. $1^{1}$ ) is a member of the crustacea and are found in most fresh water ponds. Their body is enclosed within a carapace and their length is about $1 \mathrm{~mm}$ to $3 \mathrm{~mm}$. Daphnia swims with a jerky motion through the water as the powerful 2nd antennae are thrust downward.

Ecologically, Daphnia is extremely important in the food chains of ponds and lakes. So a systematic investigation of their individual and collective behavior is

\footnotetext{
${ }^{1}$ The photograph is reprinted with the permission of Stephen Durr, http://www .

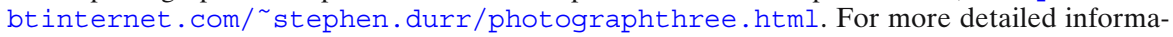
tion about Daphnia, see e.g. http://www.lander.edu/rsfox/310DaphniaLab.html, http://ebiomedia.com/gall/classics/Daphnia/
} 


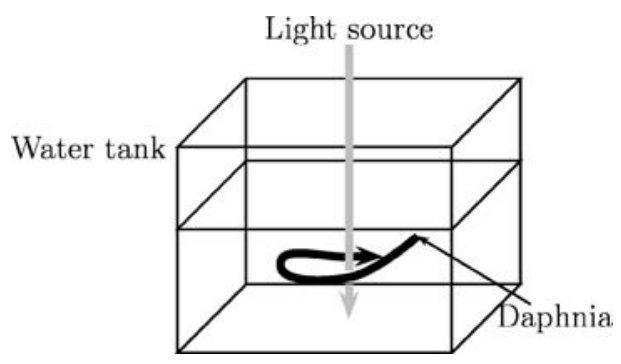

Fig. 2 Sketch of the Daphnia experiments carried out by Ordemann et al. (Ordemann, 2002; Ordemann et al., 2003b,a).

of great interest. Ordemann et al. (Ordemann, 2002; Ordemann et al., 2003a,b) have experimentally investigated the motion of Daphnia close to a vertical light shaft in both low density and high density Daphnia swarms, as we summerize in the following.

Using the experimental outline shown in Fig. 2, it was found that a single Daphnia is attracted to the light and starts to cycle (i.e. rotate) around the artificial light source, keeping its cycling direction for quite a while. In repeated experiments, however, the cycling direction may change to the opposite, which leads to the conclusion that single Daphnia, while rotating around the light beam, do not have a preferred direction of motion.

The same behavior has been observed for Daphnia swarms of sufficiently low animal density, where individual animals close to the light shaft cycle in both directions around the light shaft, frequently changing their cycling direction.

Interestingly, the situation changes if instead of single or few Daphnia a large number of animals is put in the water tank. In this case, the Daphnia start again with their cycling motion, but then all tend to move into the same direction of motion. From a physical perspective, a symmetry break is observed, i.e., the symmetry between the two possible cycling directions (left, right rotation) is clearly broken toward one of the possibilities (left or right rotation). Both of these possibilities have the same chance to occur, but only one of them is eventually realized. The vortex formation as well as the symmetry break in the cycling direction are clearly self-organized phenomena that result from the collective interaction of many animals. In order to understand this in more detail, we derive a multi-agent model in the following.

\section{Agent model of biological motion in an environmental potential}

Our modeling approach is based on active Brownian particles or Brownian agents, respectively (Schweitzer, 2003). Each of these agents is described by three state variables: spatial position $\boldsymbol{r}_{i}$, velocity $\boldsymbol{v}_{i}$ and internal energy depot $e_{i}$. The first two state variables describe the movement of the agent and can be observed from the outside. The agent's energy depot, however, is an internal variable that considers the take-up of energy from the environment, the storage of energy and conversion 
of stored energy into energy of motion (Schweitzer et al., 1998). Provided a supercritical supply of energy from the environment, the Brownian agent is capable of active movement, e.g. in a preferred direction. The term "Brownian" refers to the fact that the agents may still be subject to fluctuations that are described by a stochastic force.

The model of Brownian agents was widely discussed in different publications (Schweitzer et al., 1998; Ebeling et al., 1999; Tilch et al., 1999; Erdmann et al., 2000; Ebeling and Schweitzer, 2003). Therefore, only the basic dynamics are summarized here. For the external variables $\boldsymbol{r}_{i}$ and $\boldsymbol{v}_{i}$, we find the equations of motion in the form of a generalized Langevin equation:

$$
\frac{d}{d t} \boldsymbol{r}_{i}=\boldsymbol{v}_{i} ; \quad \frac{d}{d t} \boldsymbol{v}_{i}=-\gamma\left(v_{i}^{2}\right) \boldsymbol{v}_{i}-\left.\nabla U(\boldsymbol{r})\right|_{r_{i}}+\sqrt{2 D} \boldsymbol{\xi}_{i}(t)
$$

Here, for the mass $m=1$ is used. Causes for the change of the variables are summarized on the right-hand side of the equations. The change of the agent's position, $\boldsymbol{r}_{i}$ is caused by the movement of the agent, described by the velocity $\boldsymbol{v}_{i}$, that in turn can be changed by three different forces, explained in the following. The first term, $\gamma\left(v_{i}^{2}\right)$, is a non-linear friction function (Erdmann et al., 2000; Ebeling and Schweitzer, 2003):

$$
\gamma\left(v_{i}^{2}\right)=\gamma_{0}-d_{2} e_{i}(t)=\gamma_{0}-\frac{d_{2} q_{0}}{c+d_{2} v_{i}^{2}},
$$

which considers the active motion of the agent. $\gamma_{0}$ is the friction coefficient known from passive Brownian motion, whereas the other terms describe the influence of the internal energy depot $e_{i}(t)$, which mainly compensates this friction. Assuming that the internal energy depot relaxes very fast into a quasi-stationary equilibrium (adiabatic approximation), we derived an expression for the quasi-stationary energy depot dependent on the characteristic parameters describing its dynamics: $q_{0}$ is the influx of energy into the internal depot, which is assumed as constant here. $c$ describes the loss of energy due to internal dissipation (metabolism), whereas $d_{2}$ describes the conversion rate of internal energy into kinetic energy. The nonlinear friction function has a zero for

$$
v_{0}^{2}=\frac{q_{0}}{\gamma_{0}}-\frac{c}{d_{2}}
$$

Active motion, i.e. $\left|v_{0}\right|>0$ becomes possible only for a certain supercritical take-up of energy from the environment, $q_{0}>c \gamma_{0} / d_{2}$.

The second term in Eq. (1), $\nabla U(\boldsymbol{r})$, is used to describe the influences of the environment. The actual motion of the agent is a compromise between its active motion-which eventually would lead it everywhere, as long as internal energy is provided-and the environmental conditions which set some restrictions on this motion. The experiments described above have used a vertical beam of light that causes an attractive force on the Daphnia, which tend to cycle around it. In order 
to cope with this, we may choose the very simple assumption of an environmental potential of the form

$$
U(\boldsymbol{r})=\frac{a}{2} \boldsymbol{r}^{2},
$$

which generates an attractive force $\boldsymbol{F}=-\nabla U(\boldsymbol{r})=-a \boldsymbol{r}$ towards the center, $\boldsymbol{r}=0$.

Eventually, the last term in Eq. (1) is a stochastic force $\boldsymbol{\xi}$ (assumed to be Gaussian white noise) of strength $D$, which describes the influence of random events on the agent's motion.

The equation of motion for the Brownian agents, Eq. (1), is formulated by using two dynamical variables, $\boldsymbol{r}_{i}(t), \boldsymbol{v}_{i}(t)$, as originally proposed by Langevin for the motion of Brownian particles. In this description, fluctuations in the environment are summarized in a stochastic force that changes the acceleration of the particles (according to Newton's law of motion). In the so-called overdamped case, one can derive from this equation the overdamped Langevin equation by assuming a quasistationary velocity, $\dot{v} \approx 0$. This results in only one equation for $\dot{r}$, where the stochastic term appears with a different prefactor, $\sqrt{2 D_{r}}, D_{r}$ being the spatial diffusion coefficient. While such an approximation is convenient for further theoretical investigations, in the following we use Eq. (1) for our computer simulations.

Figure 3 shows computer simulations for the active movement of a single agent, bound by an environmental potential, Eq. (4), as described by Eq. (1). The result clearly indicates the cyclic motion round the center, which has been also observed in single Daphnia motion, as explained above. Running the computer simulations for single agents with different initial conditions eventually results in the same

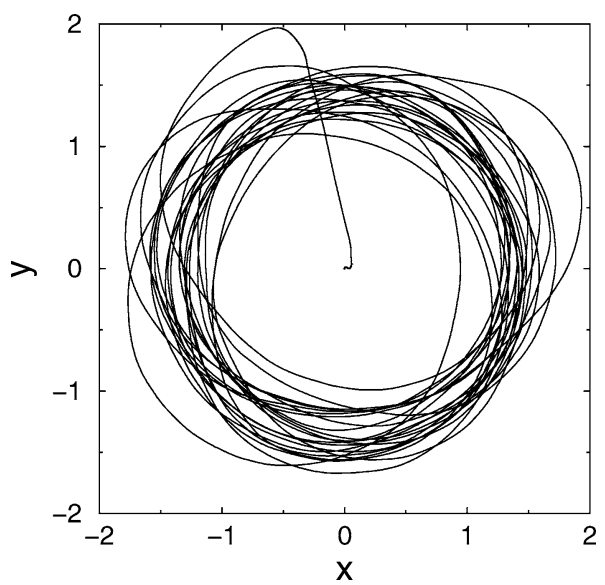

Fig. 3 Trajectory of a single Brownian agent moving in an environmental potential, Eq. (4), after $t=200$. Parameters: $\gamma=5.0, d_{2}=1.0, q_{0}=10.0, c=1.0, D=0.005, a=0.5$, i.e. supercritical take-up of energy, $q_{0}>c \gamma_{0} / d_{2}$. Initial conditions: $\{x(0), y(0)\}=\{0,0\},\left\{v_{x}(0), v_{y}(0)\right\}=\{0,0\}$, $e(0)=0$. 


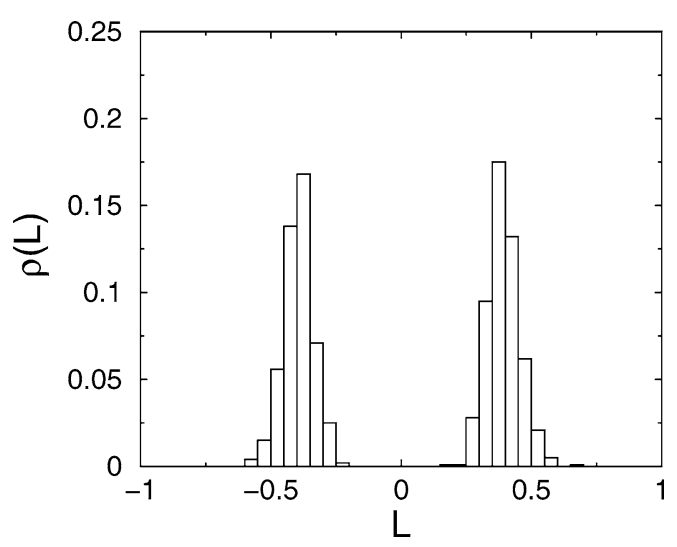

Fig. 4 Angular momentum distribution $\rho(L)$ of $N=1000$ Brownian agents after $t=150$. The positive or negative sign of $L$ indicates the right- or lefthanded rotation. Parameters: $q_{0}=10.0$, $c=1.0 \gamma=20.0, d_{2}=10.0, D=0.001, a=1.0$.

kind of cyclic motion, but with different rotational directions, i.e. left-handed or right-handed rotations. Due to stochastic influences, also changes of the direction of motion become possible. Thus, we may conclude that our model of Brownian agents sufficiently describes the observed behavior of single Daphnia.

We now turn to the case of many, i.e. $i=1, \ldots, N$ Brownian agents, which is of importance for swarming. The dynamics of the multi-agent system is then described by $2 N$ coupled (stochastic) equations of the form (1). In this case, the computer simulations shown again the characteristic rotational motion where, however, about half of the agents rotate clockwise, while the other half rotates counterclockwise. The two different cyclic directions can be clearly observed when looking at the angular momentum distribution, $\rho(L)$, where $\boldsymbol{L}$ (for $m=1$ ) is defined as $\boldsymbol{L}=\boldsymbol{r} \times \boldsymbol{v}$. As Fig. 4 shows, this is a bimodal distribution of about equal height, indicating the both left- and righthanded rotational directions with the same probability. This is not surprising as long as independent random processes with a certain symmetry are considered. But we use this graph here for comparison with the results of the elaborated model, presented in the next section.

The simulation result also does not quite agree with the observation of high density swarms of Daphnia, which apparently cycle into one, i.e. the same direction. The reasons for this mismatch are quite obvious: in our model, we have so far only considered "point-like" agents without any kind of mutual interaction, whereas in real biological systems the coherent motion of the swarm is certainly based on local interactions between the entities. The results of the present model can be compared to the case of Daphnia at low density, where cycling around the light shaft in both directions is observed (Ordemann et al., 2003b) (compare Fig. 3 with Fig. 3 of Ordemann et al. (2003a) and Fig. 4 with Fig. 1a of Ordemann et al. (2003b)). Thus, the question arises, which kind of interaction may lead to the break in the rotational symmetry, as observed in the Daphnia experiments for high animal density. 


\section{Modeling swarming with avoidance behavior}

\subsection{Interaction}

So far, different forms of global or local interactions have been introduced into swarming models. We mention

1. local interactions via a self-consistent field that has been created by the agents and in turn influences their further movement and/or "behavior" (Helbing et al., 1997; Schweitzer et al., 1997; Stevens and Schweitzer, 1997; Couzin and Franks, 2003; Erdmann and Mikhailov, 2005)—chemotactic response is a prominent example here.

2. local interactions based on the coupling of the agent's individual velocity to a local average velocity (Toner and Tu, 1995; Vicsek et al., 1995; Czirok and Vicsek, 2000; Levine et al., 2000; Grégoire et al., 2001; Couzin et al., 2002; Grégoire and Chaté, 2004).

3. global interactions, such as the coupling of the agent's individual orientation (i.e. direction of motion) to the mean orientation of the swarm (Czirok et al., 1996; Czirok and Vicsek, 2000), or the coupling of the agent's individual position to the mean position (center of mass) of the swarm (Mikhailov and Zanette, 1999; Ebeling and Schweitzer, 2001; Schweitzer et al., 2001), further couplings via the mean momentum or mean angular momentum or a combined set of invariants of motion (Czirok et al., 1996; Schweitzer et al., 2001).

4. interactions based on hydrodynamic coupling between agents (Erdmann and Ebeling, 2003).

Despite the fact that some of these models simulate coherent swarm behavior or even rotation of the swarm in the same direction, there is evidence that the underlying assumptions especially for global interactions can hardly be satisfied by biological observations, thus their biological relevance is rather questionable. Therefore, in the following section, we introduce local interactions between the agents that indeed match with biological reality. In particular, we focus on a special form of repulsive force between agents, which models avoidance maneuvers between the agents.

Experiments on Daphnia swarming (Section 2) have shown that these animals tend to cycle into the same direction for high Daphnia densities. We argue that the reason for this may be that animals try to avoid as much as possible collisions with other animals - which would occur much more frequently if different animals cycled into opposite directions at the same time. Thus, a biologically satisfactory assumption is to include avoidance behavior in our model of swarming, in order to test whether this would lead to the observed break in the rotational symmetry described above.

Daphnia are able to sense their environment to a certain degree using their sensitive mechanoreceptors (Gries et al., 1999) and their vision, i.e. they can detect animals approaching them from the fore, and then try to avoid collisions. In our models, we account for this by assuming that there is a short-ranged repulsive force between agents, to prevent their collisions. A similar idea was used by Couzin and 
Franks (2003) to describe collective motion in ants but, different from our approach, they assume a hard-core repulsion in a fixed area around each agent.

In our model, the repulsive force results from an interaction potential $V\left(r_{i}\right)$ around each agent $i$ that depends on its actual position, $\boldsymbol{r}_{i}$, and implicitly on both its actual velocity and the velocity of the approaching agent. In the following, two different ansatzes for such an avoidance potential shall be introduced. The first one discussed in the next section, has the advantage of mathematical simplicity, while the second one discussed in Section 4.3, gives smoother trajectories of the agents. Both ansatzes, however, lead to the same dynamic behavior of the swarm and therefore can be used equivalently.

\subsection{Simple avoidance model}

Our first approach is based on the assumption that the repulsion between two agents depends inversely on the Euclidian distance $r_{i j}=\left\|\boldsymbol{r}_{i j}\right\|=\left\|\boldsymbol{r}_{i}-\boldsymbol{r}_{j}\right\|$ between two agents $i$ and $j$ in two-dimensional space:

$$
V_{1}\left(r_{i j}\right)=\frac{c}{\left(r_{i j}\right)^{n}} \quad n \in \mathbb{R}^{+}
$$

where $c$ is some constant. The force between two agents $i$ and $j$ can be calculated as

$$
\boldsymbol{f}_{i j}=-\nabla V_{1}\left(r_{i j}\right)=\frac{c n}{\left(r_{i j}^{\varepsilon}\right)^{2+n}} \boldsymbol{r}_{i j}
$$

Here, we have added a small offset $\varepsilon$ to the denominator, to avoid unwanted singularities if $r_{i j} \rightarrow 0$ :

$$
r_{i j}^{\varepsilon}=\sqrt{\varepsilon+\boldsymbol{r}_{i j} \cdot \boldsymbol{r}_{i j}}
$$

In a $N$ agent system the total force on agent $i$ is simply the sum over all 2 agent forces

$$
\boldsymbol{F}_{i}=\sum_{j \neq i} \boldsymbol{f}_{i j}
$$

The consideration of the avoidance behavior leads to a modified equation of motion, i.e. Eq. (1) now reads

$$
\frac{d}{d t} \boldsymbol{r}_{i}=\boldsymbol{v}_{i} ; \quad \frac{d}{d t} \boldsymbol{v}_{i}=-\gamma\left(v_{i}^{2}\right) \boldsymbol{v}_{i}-a \boldsymbol{r}_{i}+\sum_{i \neq j} \boldsymbol{f}_{i j}+\sqrt{2 D} \boldsymbol{\xi}(t) .
$$

Again, we have assumed a linear superposition of all these forces, which seems to us the most simple assuption to start with. Other assumptions for multiple interactions are of course possible, but hardly motivated at the current stage. 


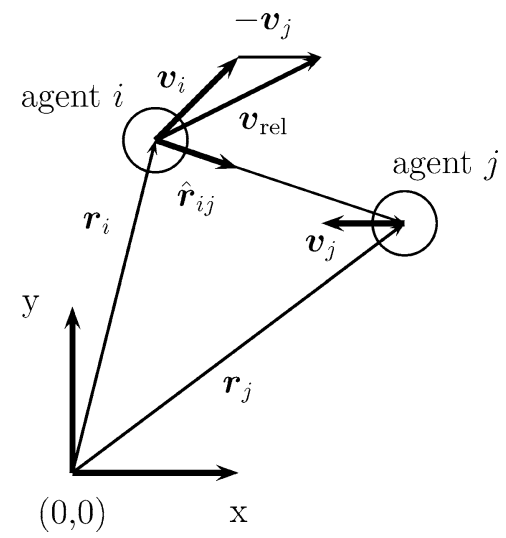

Fig. 5 Visualization of the vectorial quantities associated with the agents.

Simulations of Eq. (9) with $\boldsymbol{f}_{i j}$ defined by Eq. (6) show a swarming behavior with an angular momentum distribution as in Fig. 4. That means, we still find leftand righthanded rotation at the same time and no symmetry break. This is due to the fact that in Eq. (5) the avoidance behavior only depends on the distance between the two agents, i.e. the repulsion is the same to the front and the rear for equal distances. This assumption, however, can hardly be satisfied for Daphnia because they can mainly detect animals in front of them using their eye and the mechanoreceptors at their swimming antennas. That means that the direction of motion given by the velocity $\boldsymbol{v}_{i}$ is crucial.

To account for this, we extend Eq. (6) by multiplying our force with an asymmetry factor $\omega_{i j}$ that depends on the positions $\boldsymbol{r}_{i}, \boldsymbol{r}_{j}$ and the velocities $\boldsymbol{v}_{i}, \boldsymbol{v}_{j}$ of the two agents $i$ and $j$ as explained in the following.

The prefactor $\omega_{i j}$ has to reflect two circumstances: (i) it must increase with increasing relative velocity

$$
\boldsymbol{v}_{\text {rel }} \equiv \boldsymbol{v}_{i j}=\boldsymbol{v}_{i}-\boldsymbol{v}_{j},
$$

(see Fig. 5), because the agents would reach the point of presumable collision faster and therefore the force to avoid this has to be stronger; (ii) it has to consider whether two agents detect each other or not. This is determined by the scalar product of the relative velocity, $\boldsymbol{v}_{\text {rel }}$, and the unit vector $\hat{\boldsymbol{r}}_{i j}=\frac{\boldsymbol{r}_{i j}}{\left\|\boldsymbol{r}_{i j}\right\|}$ pointing from agent $i$ towards agent $j$ (see Fig. 5). Two agents $i$ and $j$ detect each other only if

$$
\boldsymbol{v}_{\text {rel }} \cdot \hat{\boldsymbol{r}}_{i j}>\eta \geq 0,
$$

where $\eta$ accounts for the angle of perception, i.e. $\eta=0$ would mean a visual angle of $180^{\circ}$ and $\eta>0$ corresponds to a smaller one. To avoid singularities in $\hat{\boldsymbol{r}}_{i j}$, we eventually replace $\left\|\boldsymbol{r}_{i j}\right\|$ by Eq. (7). Then, the prefactor $\omega_{i j}$ reads in its final form:

$$
\omega_{i j}^{\varepsilon}= \begin{cases}\boldsymbol{v}_{\text {rel }} \cdot \hat{\boldsymbol{r}}_{i j}^{\varepsilon}, & \text { if }\left(\boldsymbol{v}_{\text {rel }} \cdot \boldsymbol{r}_{i j}\right) \geq \eta, \quad \eta \geq 0, \\ \eta, & \text { else }\end{cases}
$$


where

$$
\hat{\boldsymbol{r}}_{i j}^{\varepsilon}=\frac{\boldsymbol{r}_{i j}}{r_{i j}^{\varepsilon}}
$$

Considering the prefactor, the avoidance term $\boldsymbol{f}_{i j}$, Eq. (6) reads now:

$$
\boldsymbol{f}_{i j}=\omega_{i j}^{\varepsilon} \cdot \frac{c n}{\left(r_{i j}^{\varepsilon}\right)^{2+n}} \boldsymbol{r}_{i j} .
$$

The equations of motion are still given by Eq. (9). Computer simulations of this extended model Eqs. (9), (14), (12) will be shown in Section 5.

\subsection{Advanced avoidance model}

Our second approach is motivated by the observation that computer simulations of the previous model, while showing the correct dynamic behavior, have the visual disadvantage of abrupt turning maneuvers of the agents. In order to improve the visual appearance, we adopt an ansatz for the avoidance potential that has been originally used to model the movement of pedestrians (Molnár, 1995):

$$
V\left(R_{i}\right)=p \cdot \exp \left(-\frac{R_{i}\left(\boldsymbol{r}_{i j}, \boldsymbol{v}_{i}, \boldsymbol{v}_{j}\right)}{\sigma}\right),
$$

$p$ denotes the strength and $\sigma$ the range of the potential, the latter being a measure of the range of detection. $R_{i}$ is a specific function of the distance between agents, as explained in the following. Since all agents are moving, agent $i$ needs to account for the space that will be occupied by all other agents $j$ in the vicinity during the next time step. This space needed, depends both on the agent's positions $\boldsymbol{r}_{j}$ and their velocity of motion, $v_{j}$, so $R_{i}$ is a function of these. For further specification, we introduce the unit vector in the direction of motion of agent $i, \boldsymbol{n}_{i}^{0}=\boldsymbol{v}_{i} /\left\|\boldsymbol{v}_{i}\right\|$; $\boldsymbol{n}_{j}^{0}$ is defined similarly. This allows to define a new velocity-dependent coordinate system for agent $i$, namely $\boldsymbol{y}_{i}$ and $\boldsymbol{x}_{i}$ defined by:

$$
\boldsymbol{y}_{i}=\frac{v_{i} \boldsymbol{n}_{i}^{0}-\delta v_{j} \boldsymbol{n}_{j}^{0}}{\left\|v_{i} \boldsymbol{n}_{i}^{0}-\delta v_{j} \boldsymbol{n}_{j}^{0}\right\|} ; \quad \boldsymbol{x}_{i} \perp \boldsymbol{y}_{i} \quad \text { and } \quad\left\langle\boldsymbol{x}_{i}, \boldsymbol{x}_{i}\right\rangle=1 .
$$

If $\delta>0$, the direction of motion of agent $j$ is also taken into account for agent $i$. The $\boldsymbol{x}_{i}$ can be constructed by the orthonomalization algorithm by GRAMSchmidT. Using this coordinate system, the dependence of $R_{i}$ on the position and velocity of agent $j$ is now given as

$$
R_{i}=\sqrt{\left\langle\boldsymbol{r}_{i}-\boldsymbol{r}_{j}, \boldsymbol{x}_{i}\right\rangle^{2}+\beta^{2}\left\langle\boldsymbol{r}_{i}-\boldsymbol{r}_{j}, \boldsymbol{y}_{i}\right\rangle^{2}}
$$

with a velocity-dependent function:

$$
\beta= \begin{cases}\beta^{\prime}: & \left\langle\boldsymbol{r}_{i}-\boldsymbol{r}_{j}, \boldsymbol{y}_{i}\right\rangle \geq 0 \\ \frac{\beta^{\prime}}{1+\lambda \cdot v_{i}}: & \left\langle\boldsymbol{r}_{i}-\boldsymbol{r}_{j}, \boldsymbol{y}_{i}\right\rangle<0\end{cases}
$$



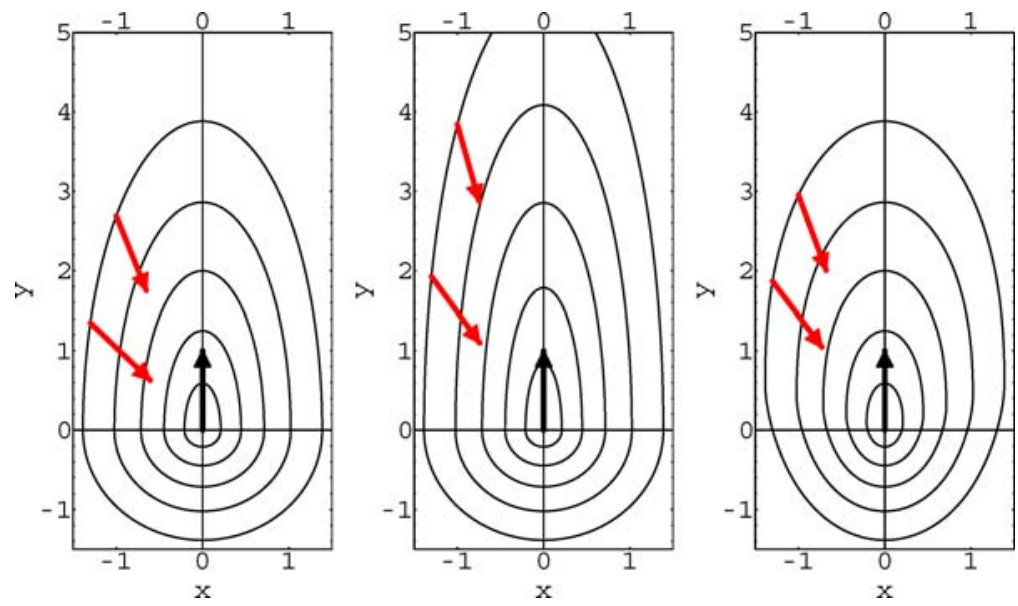

Fig. 6 Equipotential lines of the repulsive potential $V\left(r_{i}\right)$, Eq. (15) for different parameters $\delta$ and $\lambda$. Left: $\delta=0, \lambda=1.8$, Middle: $\delta=0, \lambda=3$, Right: $\delta=0.4, \lambda=1.8$. The black arrow (in the center) indicates the agent in the origin, having a velocity of $v=\{0,1\}$. The two red (gray) arrows represent other agents with the same absolute value of the velocity (1) and point towards the origin. In the left, middle and right part of the figure, the equipotential lines shown correspond to the same values for the potential.

In order to understand the meaning of the parameters $\beta^{\prime}$ and $\lambda$ we note that, if $\left\langle\boldsymbol{r}_{i}-\boldsymbol{r}_{j}, \boldsymbol{y}\right\rangle \geq 0$ then agent $i$ is moving away from agent $j$. This means that increasing the value of $\beta^{\prime}$ also means increasing $R_{i}$. Therefore, for both cases mentioned in Eq. (18), $\beta^{\prime}>1$ will lead to a reduction in the repelling force between agents $i$ and $j$. On the other hand, an increase in $\lambda \cdot v_{i}$ means that the repelling force between agents $i$ and $j$ will increase if agent $i$ is moving towards $j$. We note that these assumptions lead to an asymmetric repulsive potential $V\left(r_{i}\right)$ around each agent. The potential defined by Eq. (15) with Eq. (17) can be seen in Fig. 6 for different parameters.

Eventually, with the known repulsive interaction potential $V\left(r_{i}\right)$, the force between any two agents $i$ and $j$ is given as:

$$
\boldsymbol{f}_{i j}=-\nabla V\left(R_{i}\right)=\frac{p}{\sigma \cdot R_{i}} \exp \left(-\frac{R_{i}}{\sigma}\right)\left(\boldsymbol{r}_{i}-\boldsymbol{r}_{j}\right) .
$$

The dynamics of this model is also described by Eqs. (2), (9).

\section{Results of computer simulations of both models}

\subsection{Simulation of vortex formation}

The computer simulations of both, the simple avoidance model (Section 4.2) as well as the advanced avoidance model (Section 4.3) show indeed the expected symmetry break for the swarming behavior in agreement with the biological observations. 

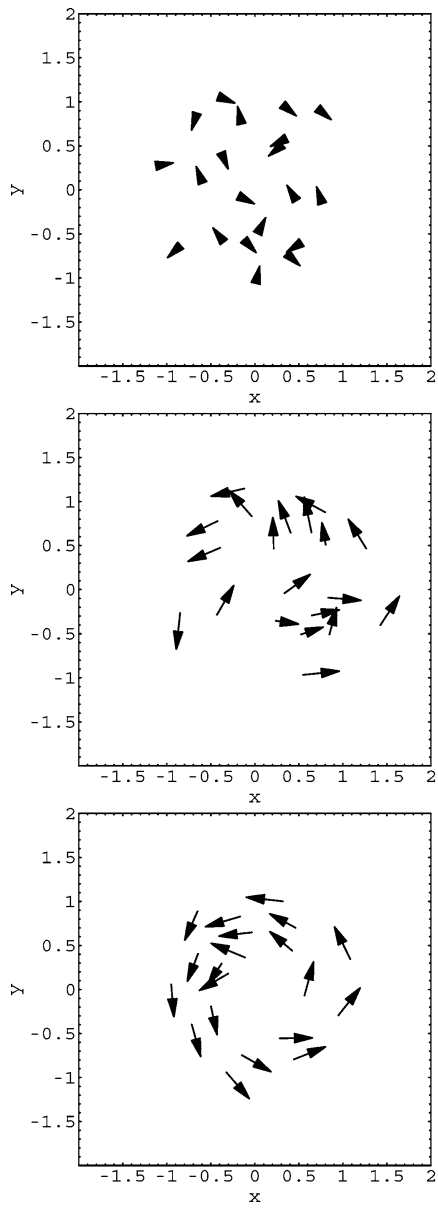

(a)

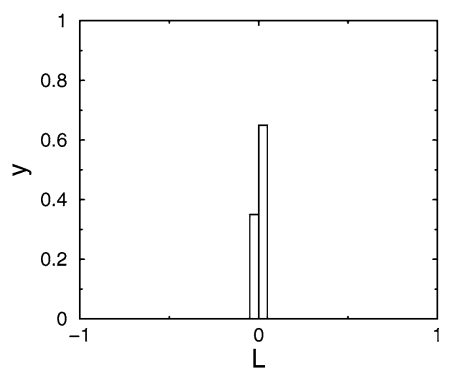

(b)

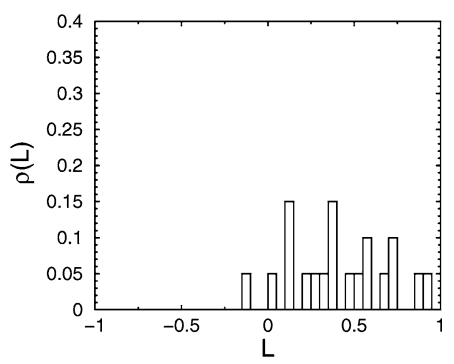

(c)

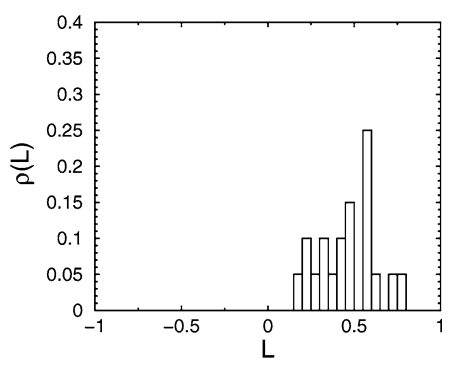

Fig. 7 Spatial snapshots (left) and distribution of angular momentum $\rho(L)$ (right) for a multiagent system $(N=20)$ at three different times: (a) $t=0$ (b) $t=8$ and (c) $t=55$. The length of the arrows indicates the velocities. Initial conditions: $\left\{x_{i}(0) ; y_{i}(0)\right\} \in[-1.5 ;+1.5],\left\{v_{i}^{x}(0), v_{i}^{y}(0)\right\}=$ $\{0,0\}, e_{i}(0)=0$, parameters: $\gamma=20.0, d_{2}=20.0, q_{0}=10.0, c=1.0, D=0.005, a=1.0 p=$ $0.8, \sigma=0.1, \delta=0.0, \lambda=10.0, \beta^{\prime}=2$. A video of the computer simulations can be viewed at http://intern.sg.ethz.ch/publications/2005/web-ms.html.

Spatial snapshots of a computer simulation of the multi-agent system with respect to avoidance behavior, together with the respective distribution of the angular momenta $\rho(L)$ are shown in Fig. 7. The results of both models can be concluded as follows:

1. On the spatial level, we observe the emergence of a coherent motion of the multi-agent swarm out of a random initial distribution. This collective motion is characterized by a unique cycling direction (either left- or righthanded rotation).

2. We further observe the formation of a vortex which is rather similar to the Daphnia swarm cycling round the light beam. 
3. While in one simulation all agents cycle in the same direction, we note that in different simulations the cycling direction can be also opposite, i.e. there is no preferred cycling direction for the swarm, which also agrees with the observations of the Daphnia swarm.

The computer simulations for the simple avoidance model, while showing the correct dynamic behavior, have the visual disadvantage of abrupt turning maneuvers of the agents. To this end, the advanced avoidance model was introduced in Section 4.3. From the computer simulations of the advanced avoidance model the following improvements can be seen:

1. The movements of the agents look smoother, more Daphnia like.

2. Although cycling in the same direction, there are some agents that come near the center.

3. For certain parameters a spontaneous change in the rotating direction can be observed. This occurs in particular if agent $i$ takes strongly into account $(\delta \approx$ 0.5 ) the movement of agent $j$.

In order to demonstrate the influence of the avoidance interaction on the collective motion of the swarm, we have conducted a computer simulation where the interaction between agents is "switched on" at time $t=150$. Thus, in the beginning, the swarm consists of non-interacting agents as described in Section 3. In Fig. 8 the evolution of the angular momentum distribution in time is shown. In the very beginning, we find a broad distribution of $\rho(L)$ centered around $L=0$. This distribution evolves towards a clear bimodal distribution as also shown in Fig. 4, indicating the complete symmetry between lefthanded and righthanded rotational

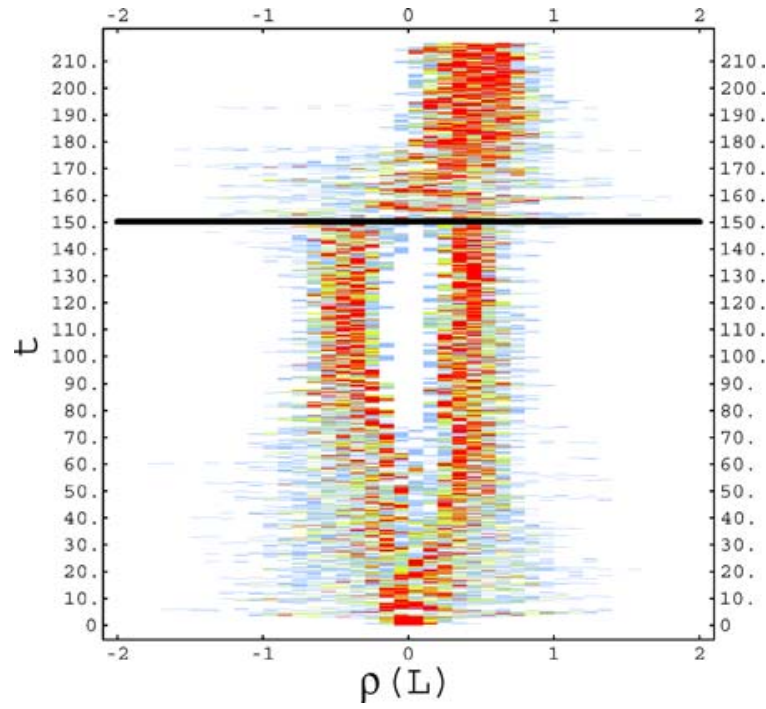

Fig. 8 Density plot (indicated by the gray scale) of the angular distribution $\rho(L)$ versus time. The interaction between agents is turned on at $t=150$ (compare to Fig. 4). For the parameters and the setup see Fig. 7. 
direction. When the interaction potential becomes effective at $t=150$, the agents start to avoid collisions and thus tend to move into the same direction. This can be clearly seen in Fig. 8 where $\rho(L)$ transforms from a bimodal into an unimodal distribution after $t>150$. The transformation period $(\Delta t \approx 30)$ is characterized by large fluctuations that sometimes even give the less frequent rotational direction a chance to take over.

The symmetry break towards one cycling direction can be also interpreted as a process of consensus formation in groups of animals as discussed by Couzin et al. (2005). They have developed a model to investigate the effective leadership and decision making in animal groups in detail. To this end, it was assumed that a small portion of the animals is informed and therefore has a desired direction of motion. The model is based on a mechanism with different tunable parameters, namely the number of informed group members and the weight they give to their preferred direction. Through extensive computer simulations the authors find that (a) for a given group size the accuracy of group motion increases asymptotically as the proportion of the informed individuals increases, and (b) the larger the group size, the smaller the proportion of informed individuals needed. Couzin et al. (2005) also investigate how individuals achieve consensus about the majority direction in case of different informed groups with competing directions. A feedback mechanism about adjusting the weights is proposed, to resolve this conflict.

We like to point out that our model does not need such tunable model parameters to achieve consensus about the moving direction. In fact, each individual in our model, while moving, has only the desire to avoid collisions with other animals. Thus, the collective motion emerges as a self-organized phenomenon. To show that the transition from bimodal to unimodal motion is inherent in our model, in the following section we show how this transition occurs with increasing swarm size.

\subsection{Swarm size dependence of vortex formation}

So far, we have shown that our model can in fact reproduce the observed cycling behavior of Daphnia swarms. This means our proposed avoidance behavior on the microscopic scale indeed leads to the symmetry break on the macroscopic scale, whereas for single agents a symmetric distribution of the angular momentum is found, in agreement with the biological observation of Daphnia.

This leads us to the question whether there is a critical swarm size at which the emergence of a vortex can be observed. In order to investigate this, we have conducted extensive computer simulations of our model with a fixed set of parameters, but different swarm sizes. We point out that the realization of a vortex swarm strongly depends on the parameters of the model, in particular how much the symmetry break is enforced by the avoidance potential. Thus, any conclusion about a critical swarm size for vortex formation, drawn in the following, is valid only for the particular parameter setting. This holds also for the scaling relation discussed below.

Secondly, we note the strong dependence of the vortex formation on stochastic influences. I.e., whether or not a vortex is formed, how long it takes for the establishment of a common cycling direction, and what this direction will be, is affected 


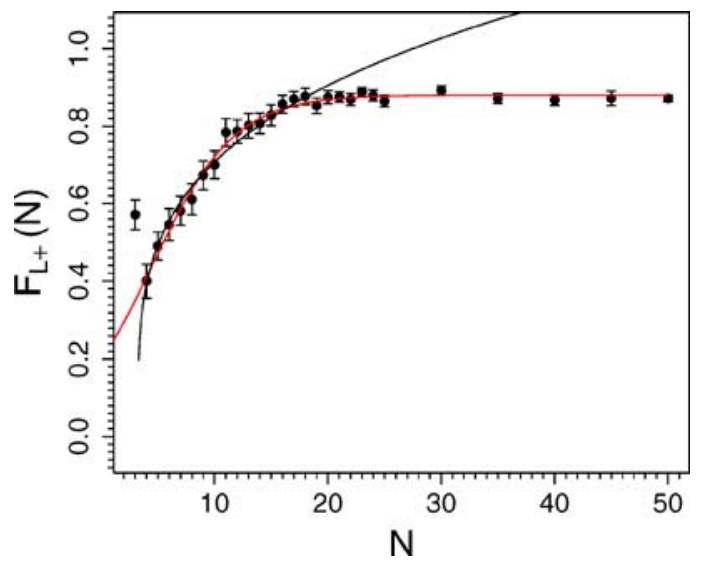

Fig. 9 Mean fraction of agents with positive angular momentum $\mathcal{F}_{L+}(N)$, Eq. (20), vs. swarm size $N$. The results are averaged over 80 simulations for each data point. The fitted curves are given by Eqs. (21), (22) (black) and Eqs. (23), (24) (red: saturation curve). Parameters: $q_{0}=10.0$, $c=1.0 \gamma_{0}=20.0, d_{2}=10.0, D=0.001, a=1.0, p=0.1, \sigma=0.1, \delta_{\alpha}=0.01, \lambda=10.0$.

by stochastic fluctuations, which play a considerable role especially for small numbers of agents.

To compensate for this, we have measured the angular momentum distribution of the swarm only after a sufficient time, $t=300$, where a common cycling direction was established in all cases. This, however, does not mean that all agents follow the same direction at that particular time. Further, there could still be large fluctuations afterwards (as can be clearly observed in the computer simulation video mentioned in Fig. 7). To account for this, we have monitored the angular momentum distribution over the next 50 time units, i.e. between $t=300-350$, and have averaged over that time interval. We note that $t=300$ does not mean simulation steps, but physical time, where the simulation interval was chosen as $\Delta t=5 \times 10^{-4}$, i.e. $t=300$ corresponds to $6 \times 10^{5}$ simulation steps and the distribution was averaged over the next $10^{5}$ simulation steps. It is obvious that due to the pairwise interaction of the agents by means of the avoidance potential, the computational effort for each simulation step also increases with $N^{2}$. Further, we have averaged the results of computer simulations over 80 runs, from which we calculated the sample standard deviation.

Figure 9 shows a function of the mean fraction of agents with a particular angular momentum dependent on the swarm size $N$,

$$
\mathcal{F}_{L+}(N)=\left|\bar{x}_{L+}-\bar{x}_{L-}\right|=\left|2 \bar{x}_{L+}-1\right| ; \quad \bar{x}_{L+}=\frac{1}{s m N} \sum_{k=1}^{s} \sum_{n=0}^{m} N_{L+}^{(k)}(t+n \Delta t),
$$

where $N_{L+}^{(k)}(t)$ is the number of agents found with a positive angular momentum in simulation $(k)$ at time $t$. For the simulations $s=10, t=300, \Delta t=5 \times 10^{-4}$ and $m=10^{5}$ were chosen. 
If a swarm has a bimodal angular momentum distribution as shown in Fig. 4, $\bar{x}_{L+}$ would be 0.5 and $\mathcal{F}_{L+}(N)=0$, i.e. no common cycling direction has been established, that is followed by a majority of agents. On the other hand, a clear unimodal distribution as e.g. observed in Fig. 7, would lead to either $\bar{x}_{L+}=0$ or 1, i.e. $\mathcal{F}_{L+}(N)=1$.

As Fig. 9 indicates, the emergence of a common cycling direction occurs between swarm sizes of 4-18 agents (for the given set of parameters). Even for large swarm sizes, the common cycling direction is not followed by all agents, a noticeable minority fraction still cycles its own way. This, however, should be not considered as a drawback of the model, in fact it makes it much more realistic, as the computer simulation video also shows. This behavior also agrees with observations of Daphnia swarms at high density. In particular, animals at the border of the swarm still do not follow the cycling direction of the majority.

We note that the occurence of a common cycling direction is not an abrupt transition, but a gradual one. One can argue that this resembles a kinetic phase transition analogous to the continuous phase transition in equilibrium systems (Vicsek et al., 1995), i.e.

$$
F_{L+}(N)=c_{0}\left(N-N_{c}\right)^{\kappa},
$$

where $N_{c}$ is the onset of the transition and $\kappa$ is the critical exponent. For their model Vicsek et al. (1995) have determined $\kappa$ as 0.35 . We have tested this finding against our simulation results, as shown in Fig. 9 and found for the least square fit and standard errors in the region $N \in[4,20]$ :

$$
N_{c}=3.240 \pm 0.328 ; \quad c_{0}=0.422 \pm 0.029 ; \quad \kappa=0.270 \pm 0.025 \text {. }
$$

On the first glimpse, there is a close similarity between Vicsek et al. (1995) and our findings with respect to the value of the critical exponent $\kappa$. I.e., we emphasize that vortex formation under repulsion also occurs at a critical point.

However, as Fig. 9 clearly shows, the scaling suggested by Vicsek et al. (1995) only holds for a restricted range of $\mathrm{N}$-which by the way was already the case in the original paper (cf. Fig 2b of Vicsek et al. (1995)). A much better fit of the observed transition is provided by the following function:

$$
F_{L+}(N)=\kappa\left[1+\exp \left(-\frac{N-N_{c}}{\tau}\right)\right]^{-1},
$$

with the best-fit parameters and standard errors:

$$
N_{c}=4.496 \pm 0.136 ; \quad \tau=3.651 \pm 0.161 ; \quad \kappa=0.880 \pm 0.004 .
$$

As shown in Fig. 9, this function fits the simulated data extremely well. The only outlier for $N=3$ is due to the fact, that for 3 agents at least 2 agents go in the same direction. That means that the probability of breaking the symmetry is a lot higher in this particular case. 
In addition to the depencence on the swarm size or density, Vicsek et al. (1995) also discussed a phase transition with respect to noise, $\eta$, in the alignment of the individuals and suggested a scaling similar to Eq. (21). As the critical value, $\eta_{\mathrm{c}}$, depends on the lattice size of their computer simulations, there is no direct comparison with this result possible. However, we note the obvious similarities to other types of structure formation which also occur only below a certain critical temperature. This holds also for our model where a common cycling direction can emerge only below a critical noise level. As a difference to the investigations of Vicsek et al. (1995), noise in our model enters the equation of motion, Eq. (9), whereas the avoidance interaction is without noise.

\section{Model testing of avoidance maneuvers}

So far, reasonable assumptions about local interactions of Daphnia (such as local repulsion) have been taken into account. But it still remains to be tested empirically and theoretically whether avoidance maneuvers of Daphnia are really responsible for the symmetry break observed in high density swarms.

To gather evidence on the microscopic dynamics of our model, we used data provided by Anke Ordemann (Ordemann et al., 2004) about avoidance maneuvers of pairs of Daphnia approaching each other in a horizontal plane. Snapshots of a typical avoidance time series are shown in Fig. 10. A thorough analysis of similar experiments would allow to determine the parameters in our model, but the time series presented was the only data made available to us. Nevertheless, this sequence already enables us to qualitatively compare the experimental findings with our theoretical model of avoidance behavior.

To this end, we have simulated the avoidance maneuver of two agents in a situation similar to the one shown in Fig. 10. From the simulation results shown in the left part of Fig. 11 it can be clearly seen that the two agents avoid each other not in

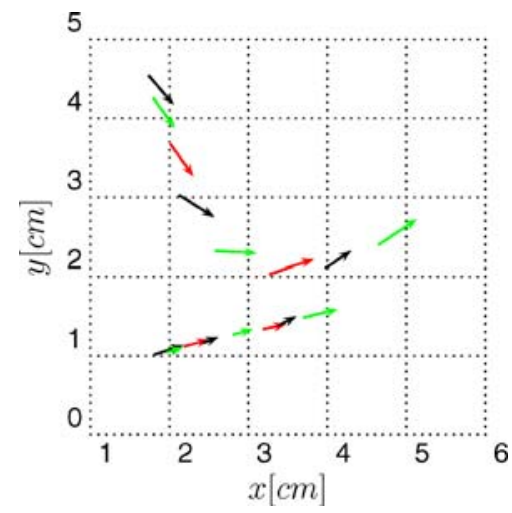

Fig. 10 Experimental observation of an inter-animal avoidance maneuver of two Daphnia in the horizontal plane (Ordemann et al., 2004). In our representation of the available data, each arrow represents the spatial orientation (head and tail) of the Daphnia at successive times (every $0.2 \mathrm{~s}$ ). In the sequence, same arrow colors (gray scales) correspond to the same time. 

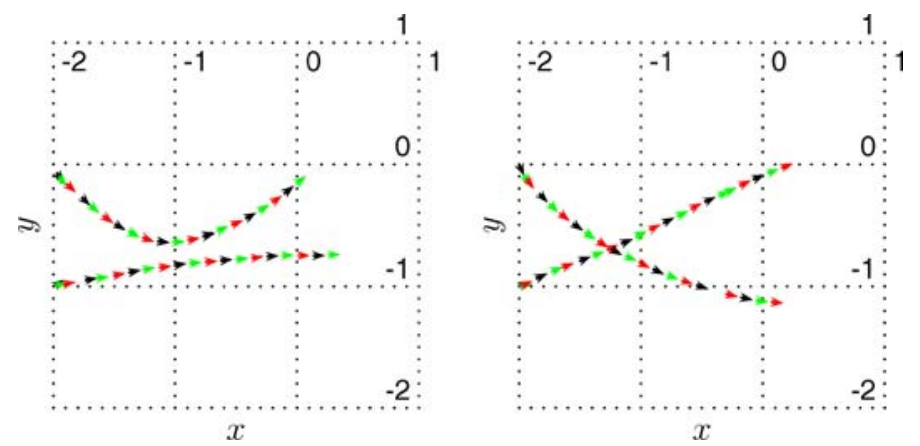

Fig. 11 Left: Simulation of an inter-agent avoidance maneuver in the horizontal plane. Each arrow represents the spatial orientation (drawn from the velocity vector) of the agent at successive times (every 0.2 time units). In the sequence, same arrow colors (gray scales) correspond to the same time. Parameters: $q_{0}=10.0, c=1.0 \gamma_{0}=20.0, d_{2}=10.0, D=0.001, p=0.2, \sigma=0.2, \delta_{\alpha}=$ $0.1, \lambda=10.0, a=0.1$, Right: Simulation with the same setting but without avoidance interaction, $p=0.0$.

a symmetrical way, but rather similar to the experimental findings. One may argue that this is due to the additional influence of the environmental potential which may more affect the agent closer to the origin $(0,0)$. In order to test this, we have simulated the same situation without the avoidance interaction potential, but just the environmental potential. The result, shown in the right part of Fig. 11, clearly indicates that the environmental potential has only very little affects the agent's trajectories and thus cannot be responsible for the realistic avoidance behavior shown in the left part of Fig. 11.

Comparing the simulation of the model with the above experimental observations in Daphnia, it becomes obvious that inter-animal avoidance maneuvers similar to the ones incorporated in the current Brownian agent model are most likely involved in the mechanism that causes symmetry breaking for high Daphnia density and leads to the observed vortex swarms.

\section{Discussion}

In this paper, we tried to understand the vortex swarming behavior observed in Daphnia from rather minimalistic assumptions that, however, should have a clear biological relevance. To this end, we introduced a multi-agent model based on the concept of Brownian agents. Different from other modeling approaches which are based e.g. on cellular automata, our model considers both continuous time and space. Further, in addition to external and interaction forces effecting the agent's behavior, we consider stochastic influences resulting e.g. from random events or fluctuations in the environment. As a difference to multi-agent models developed in artificial life, our model further allows for a more specific discussion of the different parameters affecting the agent's motion. It can be also seen as an alternative to some of the self-propelled particle models (Vicsek et al., 1995; Grégoire et al., 2001; Grégoire and Chaté, 2004) where alignment between individuals is 
explicit. In our model, we demonstrate that vortex swarming does not require an explicit alignment rule for individuals but can be achieved through a combination of attraction and repulsion. This insight allows us to reduce assumptions about the cognitive requirements of swarming organisms.

At the end, we give a critical discussion of the Brownian agent model in the biological context of Daphnia swarming, in order to point out its limitations and to give some hints for experimental verifications.

The equation of motion for the Brownian agents is a generalized Langevin Eq. (1), which models the motion of the animals as continuous in space and time. The movement of single Daphnia, however, consists of a series of jumps as can be also observed in experimental observations. This can at least be partially covered by using a larger time step in our simulation, resulting in larger jumps during each time interval. We argue that this would not change the overall dynamic behavior of our model. Thus, we kept the continuous approximation of the discrete jumps and the small time step to avoid numerical instabilities.

Our theoretical description is based on the assumption of a quasistationary energy depot of the agents. This implies a constant take-up of energy, $q_{0}$, whereas Daphina feed in cycles while they do not move actively. Thus, a switch-like change in behavior seems to be more appropriate. This can be covered in our model by assuming the take-up rate of energy a time-dependent function, $q(t)$, and replacing the continuous acceleration term in Eq. (1), $d_{2} e_{i}(t)$, by a more complex term that reflects a switch-like change between starvation and active motion.

Response to external forces and force-based interaction between the agents are two basic ingredients of our model of Brownian agents. Interaction of biological entities, on the other hand, is often driven by internal and behavioral reasons. This certainly sets the limits for our approach which is inspired by, and based of, physical considerations using minimal assumptions. Physics, per se, has no concept of "behavior" based on internalized motivations of an agent. Thus, in our model every kind of internal "driving forces" has to be externalized by assuming that the agent behaves as it would follow an external force that leads it to the desired behavior. Such an approach does not claim that these forces really exist in the outer world, it is rather a convenient modeling formalism that allows to apply the concepts of physics to the much more complex behavior of animals. An alternative could be concepts from artificial intelligents, where the internal dynamics of agents is modeled explicitely (the so-called BDI (belief-desire-intention) agents, for example, can have their own internal world view).

In our model of Brownian agents, we could demonstrate the emergence of a vortex swarm based on local asymmetric interactions of the agents. The global dynamic behavior was also found for other models (see also Section 4.1) exploiting other mechanisms of symmetry breaking. We do not want to argue here about the most simplest one-at the end, one has to find a compromise between "simple" and biologically "satisfied". Many of the proposed mechanisms-such as the hydrodynamic coupling - still lack an experimental justification. Our avoidance model, on the other hand, could be at least visually tested by comparing the experimental observations of two Daphnia approaching each other with a similar situation from the computer simulations. 
Noteworthy, in our model the emergence of a vortex swarm is not enforced by the alignment of the agents, as used in other models. Instead, we have included only the simple assumption of collision avoidance. The specific form of our avoidance potential penalizes mainly the head-head collision and thus promotes the dispersion of the agents (and not the alignment). As a consequence, to obtain a vortex swarm, an additional attraction force is needed, which in the considered case results from the environmental potential (attraction towards the center). This is not a drawback of the model, but justified by the real experimental observation. As shown by Schweitzer et al. (2001), under certain circumstances the effect of an external parabolic potential is also equivalent to an attractive force between the agents. The emergence of a vortex swarm can be seen as a dynamic compromise between three different requirements: active motion (to keep the agents moving), asymmetric repulsive forces (e.g. to avoid collisions) and attractive forces (either enforced by a local agent-agent attraction, or an external potential). While the first two requirements alone would simply lead to a dispersion, the latter one results in a compression (or confinement) of the swarm. From a physics perspective the vortex swarm is a stable attractor of the multi-agent dynamics; from an "economics" perspective one could think of global utility maximization balancing out all individual requirements (such as avoiding discomfort from both collisions and separation).

A noticeable advantage of our model is that it does not break down for small swarm sizes, so it can be used to simulate both single animals and swarms of low and of high density, whereas other models mainly concentrate on the dynamics of reasonably sized swarms. This allows in particular to investigate the transition from the uncorrelated (bi-directional) rotation of single agents to the correlated vortex formation of the swarm, as shown in Fig. 9.

So far, we have shown a qualitative agreement between our computer simulations and some experimental observations in Daphnia. A quantitative verification, or even a prediction of Daphnia behavior under different real conditions is still missing. In this respect, our model is not different from other theoretical models proposed. A first step towards verification involves the experimental determination of the parameters. In this paper, we can just propose some ideas:

- Neglecting the jump-like motion of single Daphnia and using the approximation of a (quasi-)stationary velocity, one should be able to estimate an average velocity of cycling Daphnia. This can be directly related to the stationary velocity $v_{0}$, Eq. (3), that enters the equation of motion used in the model.

- The parameters determining the avoidance behavior may be estimated by a direct comparison between the experimental observation, Fig. 10 and the computer simulation, Fig. 11. Obviously, the sole event shown in this paper, is not sufficient for that; so, we would expect further experimental investigations here.

- Another way to (indirectly) estimate the parameters of the avoidance potential is via the local swarm density, or the (average) spatial extension of a swarm of given size. These are determined by our model parameters and could be possibly compared with experiments (varying both the strength of the light beam and the swarm size). 
- By varying the swarm size, one can also experimentally test the onset of the vortex swarming, and compare this to the respective computer simulations shown in Fig. 9. As mentioned, the transition range towards the vortex formation in the model strongly depends both on swarm size and on the parameters characterizing the avoidance potential. So, in addition to the observation of avoidance maneuvers of two animals, this yields a macroscopic verification for the parameters of the avoidance potential.

Eventually, we want to point out some situations where the model could make predictions about Daphnia behavior which may be tested experimentally. The model uses the assumption of an environmental potential, that in the current investigation results in an attraction toward the center, this way considering the influence of the vertical light beam on the Daphnia. The real influence could be tested by producing Daphnia mutants that are insensitive to light. Then we expect no attractive force, and hence no vortex swarming, in agreement with the explanations above. Also, one could think of two different vertical light beams in the water tank, at a certain distance. Starting from a homogeneous spatial distribution of agents, the model would predict the occurrence of two different vortex swarms around the two centers, each probably having its own rotational direction, as long as the distance between the light beams is large enough. For smaller distances (where the critical distance may be also a function of the agent density in the system), we would expect from the model interferences at the boarder between the two rotating swarms, which would lead to additional couplings and thus maybe to a synchronization of the rotational directions. The situation of the two separated vertical light beams would also allow us to test whether the attraction of the Daphnia is limited by some maximum local density of the swarm, as suggested by our model. In this case, we would find in the experiments two distinct swarms, whereas without saturation effects, one could possibly find one swarm only, with a much higher density.

A remaining question is weather the model in the current form is also applicable to other species. Vortex swarming, as we have pointed out, is a widely spread phenomenon observed also in fish, or bacteria. We argue that the principle (qualitative) features of vortex swarming are covered by our model, as long as (local or global) attraction and asymmetric repulsion (e.g. via avoidance maneuvers) play a considerable role. This may hold for fish, but probably not for gliding bacteria like Paenibacillus, which also show vortex formation (Ben-Jacob, 2003). In the latter case, adhesion forces may play a much more important role as one can also deduce by looking at the sharp external boundaries of the bacterial swarm. However, it would be still possible to adapt the model of Brownian agents also for this situation by including other terms of local interaction.

\section{Acknowledgments}

The authors thank Anke Ordemann for discussion and for providing data of the Daphnia-Daphnia avoidance maneuver investigated in Section 6. 


\section{References}

Ben-Jacob, E., 2003. Bacterial self-organization: co-enhancement of complexification and adaptability in a dynamic environment. Phil. Trans. R. Soc. Lond. A 361, 1283-1312.

Caraco, T.S., Martindale, S., Pulliam, H.R., 1980. Avian flocking in the presence of a predator. Nature 285, 400-401.

Couzin, I., Franks, N., 2003. Self-organized lane formation and optimized traffic flow in army ants. Proc. R. Soc. London Ser. B 270, 139-146.

Couzin, I., Krause, J., James, R., Ruxton, G., Franks, N.R., 2002. Collective memory and spatial sorting in animal groups. J. Theor. Biol. 218, 1-11.

Couzin, I.D., Krause, J., 2001. The social organisation of fish schools. Advances in Ethology 36(64).

Couzin, I.D., Krause, J., Franks, N.R., Levin, S.A., 2005. Effective leadership and decision making in animal groups on the move. Nature 433, 513-516.

Czirok, A., Ben-Jacob, E., Cohen, I., Vicsek, T., 1996. Formation of complex bacterial colonies via self-generated vortices. Phys. Rev. E 54(2), 1791-1801.

Czirok, A., Vicsek, T., 2000. Collective behavior of interacting self-propelled particles. Physica A $281,17-29$.

Deutsch, A. (1999). Principles of morphogenetic motion: swarming and aggregation viewed as self-organization phenomena. J. Biosci. 24(1), 115-120.

Ebeling, W., Schweitzer, F., 2001. Swarms of Particle Agents with Harmonic Interactions. Theory in Biosciences 120(3-4), 207-224.

Ebeling, W., Schweitzer, F., 2003. Self-Organization, Active Brownian Dynamics, and Biological Applications. Nova Acta Leopoldina NF 88(332), 169-188.

Ebeling, W., Schweitzer, F., Tilch, B., 1999. Active brownian particles with energy depots modelling animal mobility. BioSystems 49, 17-29.

Erdmann, U., E.W., Mikhailov, A.S., 2005. Noise-induced transition from translational to rotational motion of swarms. Phys. Rev. E 71(051904).

Erdmann, U., Ebeling, W., 2003. Collective motion of brownian particles with hydrodynamic interactions. Fluctuation Noise Lett. 3, L145-L154.

Erdmann, U., Ebeling, W., Schimansky-Geier, L., Schweitzer, F., 2000. Brownian particles far from equilibrium. Eur. Phys. J. B 15(1), 105-113.

Flierl, G., Grünbaum, D., Levin, S., Olson, D., 1999. From individuals to aggregations: the interplay between behavior and physics. J. Theoret. Biol. 196, 397-454.

Grégoire G., Chaté, H., 2004. Onset of collective and cohesive motion. Phys. Rev. Lett. 92, 025702.

Grégoire, G., Chaté, H., Tu, Y., 2001. Active and passive particles: Modeling beads in a bacterial bath. Phys. Rev. E 64, 011902.

Gries, T., Jöhnk, K., Fields, D., Strickler, J., 1999. Size and structure of 'footprints produced by Daphnia: impact of animal size and density gradients. J. Plankton Res. 21, 509-523.

Grünbaum, D., Okubo, A., 1994. Modelling Social Animal Aggregation. In: Levin, S.A. (Ed.), Frontiers in Theoretical Biology. Springer, New York, vol. 100 of Lecture Notes in Biomathematics.

Hall, S.J., Wardle, C.S., MacLennan, D.N., 1986. Predator evasion in a fish school: test of a model of the fountain effect. Mar. Biol. 91, 143-148.

Helbing, D., Schweitzer, F., Keltsch, J., Molnár, P., 1997. Active walker model for the formation of human and animal trail systems. Phys. Rev. E 56(3), 2527-2539.

Huth, A., Wissel, C., 1992. The simulation of the movement of fish schools. J. Theor. Biol. 156, 365-385.

Huth, A., Wissel, C., 1994. The simulation of fish schools in comparison with experimental data. Ecological Modelling 75-76, 135-146.

Jakobsen, P., Johnsen, G., 1987. Behavioral response of the water flea Daphnia pulex to a gradient in food concentration. Anim. Behav. 35, 1891-1895.

Jakobsen, P.J., Birkeland, K., Johnsen, G.H., 1994. Swarm location in zooplankton as an antipredator defense mechanism. Anim. Behav. 47, 175-178.

Jensen, K., 2000. Gregariousness in Daphnia: significance of food distribution and predator evasion. University Bergen, Dep. of Zoology, Norway, Phd. Thesis.

Kleiven O., Larsson, P., Hobæk, 1996. Direct distributional response in Daphnia pulex to a predatorkairomone. J. Plankton Res. 18, 1341-1348. 
Kunz, H., Hemelrijk, C.K., 2003. Artificial fish schools: collective effects of school size, body size, and body form. Artificial Life 9, 237-253.

Kvam, O., Kleiven, O., 1995. Diel horizontal migration and swarm formation in Daphnia in response to Chaoborus. Hydrobiol. 307, 177-184.

Larsson, P., 1997. Ideal free distribution in Daphnia? Are daphnids able to consider both the food patch quality and the position of competitors? Hydrobiologia 360, 143-152.

Larsson, P., Kleiven, O., 1995. Food search and swimming speed in Daphnia. In: Lenz, P.H., Hartline, D., Purcell, J., Macmillan, D. (Eds.), Zooplankton: Sensory Ecology and Physiology. Gordon and Breach, pp. 375-387.

Levine, H., Rappel, W.-J., Cohen, I., 2000. Self-Organization in Systems of Self-Propelled Particles. Phys. Rev. E 63, R017101.

Lobel, P.S., Randall, J.E., 1986. Swarming behavior of the hyperiid amphipod Anchylomera blossevilli. J. Plankton Res. 8, 253-262.

Mikhailov, A., Zanette, D.H., 1999. Noise-induced breakdown of coherent collective motion in swarms. Phys. Rev. E 60, 4571-4575.

Molnár, P., 1995. Modellierung und Simulation der Dynamik von Fussgängerströmen. Aachen: Shaker. ISBN: 3-8265-1191-3.

Øien, A.H., 2004. Daphnicle dynamics based on kinetic theory: an analogue-modelling of swarming and behaviour of Daphnia. Bull. Math. Biol. 66, 1-46.

Okubo, A., Levin, S., 2002. Diffusion and Ecological Problems. New York: Springer.

Ordemann, A., 2002. Vortex-Swarming of the Zooplankton Daphnia. Biol. Physicist 2(3), 5-10.

Ordemann, A., Balazsi, G., Moss, F., 2003a. Motions of daphnia in a light field: random walks with a zooplankton. Nova Acta Leopoldina 88(332), 87-103.

Ordemann, A., Balazsi, G., Moss, F., 2003b. Pattern formation and stochastic motions of the zooplankton Daphnia in light fields. Physica A 325, 260-266.

Ordemann, A., Garcia, R., Moss, F., 2004. Avoidance maneuvers observed in Daphnia. (unpublished).

Parrish, J., Edelstein-Keshet, L., 1999. Complexity, pattern, and evolutionary trade-offs in animal aggregation. Science 284, 99-101.

Parrish, J.K., Hamner, W. (eds.), 1997. Animal Groups in Three Dimensions. Cambridge University Press, Cambridge.

Parrish, J.K., Viscido, S.V., Grünbaum, D., 2002. Self-organized fish schools: An examination of emergent properties. Biol. Bull. 202, 296-305.

Partridge, B.L., 1982. The structure and function of fish schools. Scientific American 246, 90-99.

Schweitzer, F., 2003. Brownian agents and active particles. Collective dynamics in the natural and social sciences, Springer Series in Synergetics.

Schweitzer, F., Ebeling, W., Tilch, B., 1998. Complex motion of Brownian particles with energy depots. Phys. Rev. Lett. 80(23), 5044-5047.

Schweitzer, F., Ebeling, W., Tilch, B., 2001. Statistical mechanics of canonical-dissipative systems and applications to swarm dynamics. Phys. Rev. E 64(2), 021110-1-021110-12.

Schweitzer, F., Lao, K., Family, F., 1997. Active random walkers simulate trunk trail formation by ants. BioSystems 41, 153-166.

Stevens, A., Schweitzer, F., 1997. Aggregation induced by diffusing and nondiffusing media. In: Alt, W., Deutsch, A., Dunn, G. (Eds.), Dynamics of Cell and Tissue Motion. Birkhäuser, Basel, pp. 183-192.

Tilch, B., Schweitzer, F., Ebeling, W., 1999. Directed motion of brownian particles with internal energy depot. Physica A 273(3-4), 294-314.

Toner, J., Tu, Y., 1995. Long-range order in a two-dimensional dynamical $X Y$ model: How birds fly together. Phys. Rev. Lett. 75(23), 4326-4329.

Vicsek, T., Czirok, A., Ben-Jacob, E., Cohen, I., Shochet, O., 1995. Novel type of phase transition in a system of self-driven particles. Phys. Rev. Lett. 75, 1226-1229. 